
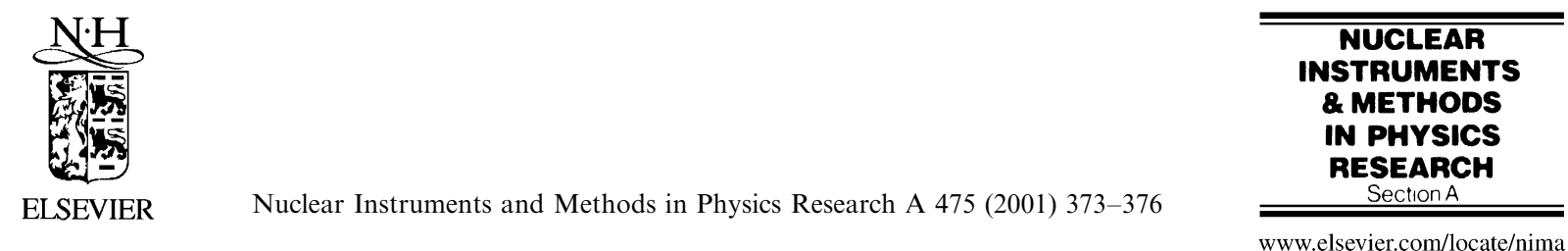

\title{
Nonlinear harmonic generation in distributed optical klystrons
}

\author{
H.P. Freund ${ }^{\mathrm{a}, *}$, G.R. Neil ${ }^{\mathrm{b}}$ \\ ${ }^{a}$ Science Applications International Corporation, 1710 Goodridge Drive, McLean, VA 22102, USA \\ ${ }^{\mathrm{b}}$ Thomas Jefferson National Accelerator Facility, Newport News, VA 23606, USA
}

\begin{abstract}
A distributed optical klystron has the potential for dramatically shortening the total interaction length in high-gain free-electron lasers (INP 77-59, Novosibirsk, 1977; Nucl. Instr. and Meth A 304 (1991) 463) in comparison to a singlewiggler-segment configuration. This shortening can be even more dramatic if a nonlinear harmonic generation mechanism is used to reach the desired wavelength. An example operating at a $4.5 \AA$ fundamental and a $1.5 \AA$ harmonic is discussed. (C) 2001 Elsevier Science B.V. All rights reserved.
\end{abstract}

PACS: 41.60.Cr; 52.75.Ms

Keywords: Free-electron lasers; Harmonics; Optical klystron

The free-electron laser (FEL) is a candidate for 4th generation X-ray light sources [1-3], and are intended to operate in the Self-Amplified Spontaneous Emission (SASE) mode where noise is amplified to high levels in a single pass. However, the gain lengths are long and the wiggler length required can exceed $100 \mathrm{~m}$. One alternative to this design is to use a distributed optical klystron employing a multi-segment wiggler in which dispersive magnetic elements are located in the gaps between the wigglers. High gain is achieved because the dispersive elements enhance the electron bunching [4-8]. The multi-segment design has been discussed in regard to interaction at the fundamental [5-8], and it is known that the bulk amplification can be much faster than exponential if a sufficiently high beam brightness can be

*Corresponding author. Tel.: + 1-202-767-0034; fax: + 1-202734-1280.

E-mail address: freund@mmace.nrl.navy.mil (H.P. Freund). achieved. Here, we discuss the possibility of using the nonlinear harmonics, and employ dipole triplets (chicanes) as dispersive elements where the outermost dipoles have the same magnitudes $\left(B_{0}\right)$ and lengths $\left(L_{\mathrm{d}}\right)$ and the center dipole has the same magnitude as the outermost dipoles but twice the length.

A unit cell of the structure is illustrated schematically in Fig. 1, and the overall structure is composed of many such unit cells. The 3D nonlinear, polychromatic simulation code MEDUSA $[9,10]$ is used. MEDUSA uses a Gaussian electron beam distribution in energy and phase space, planar wiggler geometry, and treats the electromagnetic field as a superposition of GaussHermite modes. The field equations are integrated simultaneously with the 3D Lorentz force equations for an ensemble of electrons. No wiggler average is imposed on the orbit equations; hence, MEDUSA is capable of propagating the electron beam through arbitrary magnetic structures (such 


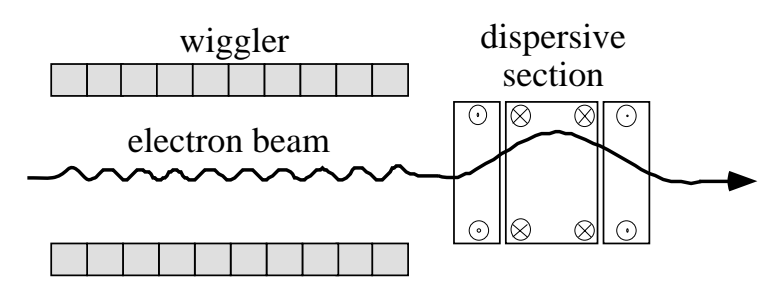

Fig. 1. Schematic illustration of a unit cell.

as wigglers, quadrupoles, dipoles, as well as FODO lattices and dipole chicanes) and includes the self-consistent interaction with the electromagnetic wave.

We consider a $1.5 \AA$ source corresponding to the Linac Coherent Light Source (LCLS) [2] which employs a $14.35 \mathrm{GeV} / 3400 \AA$ electron beam with an rms beam radius of $88 \mu \mathrm{m}$, and operates on the fundamental. Gun/linac simulations indicate that a slice emittance $\varepsilon_{n}=1.0 \pi-1.5 \pi \mathrm{mm} \mathrm{mrad}$ and an energy spread of $0.006 \%$ can be achieved. For simplicity, we assume parabolic-pole face (PPF) wiggler segments. In studying the harmonic interaction, we lower the beam energy to $8.265 \mathrm{GeV}$ to obtain a fundamental resonance at $4.5 \AA$, and reduce the length of each wiggler segment to $2.25 \mathrm{~m}$ with respect to the parameters used in Ref. [8]. All other parameters are held fixed; in particular, the wiggler segments are separated by $2.0 \mathrm{~m}$ gaps, and the dipole chicanes are centered in the gaps. The individual dipoles have lengths of $0.4 \mathrm{~m}$ (for an overall chicane of $1.6 \mathrm{~m}$ ). The number of unit cells and the dipole magnitudes are varied in simulation to obtain the optimal performance.

The length of the wiggler segments is less than the gain length for the $8.265 \mathrm{GeV} / 4.5 \AA$ (fundamental) interaction. MEDUSA is in substantial agreement with the results of linear theory [11] for a single-segment wiggler from which we find gain lengths of $2.96 \mathrm{~m}$ in MEDUSA and $2.99 \mathrm{~m}$ gain length from the linear theory. It was pointed out in treating the fundamental interaction at $1.5 \AA[8]$ that the growth in the distributed optical klystron can be substantially faster than exponential. This is because the enhanced bunching in a chicane gives rise to amplification in the next wiggler segment that is initially faster than exponential but falls off to the expected exponential rate after about one gain length. Therefore, if the wiggler segments are shorter than a gain length, then the interaction is faster than exponential and the saturation length can be considerably shortened.

The interaction in an optical klystron is very sensitive to both beam energy and the magnetic field in the chicanes because the increase in the path length due to the chicane is $L=2 L_{\mathrm{d}}^{3} B_{0}^{2} / 3 \gamma^{2} c^{2}$, so the change in path length with energy relative to the wavelength $(\lambda)$ is

$\frac{\Delta L}{\lambda}=\frac{4 L_{\mathrm{d}}^{3} \Omega_{0}^{2}}{3 \gamma^{2} c^{2} \lambda}\left(\frac{\Delta B_{0}}{B_{0}}-\frac{\Delta E_{\mathrm{b}}}{E_{\mathrm{b}}}\right)$

where $\Omega_{0}$ is the chicane cyclotron frequency. Optimal performance requires that $|\Delta L / \lambda|<1$ or else overbunching occurs. This implies that

$\frac{\Delta E_{\mathrm{b}}}{E_{\mathrm{b}}}<\frac{3 c^{2} \lambda_{\mathrm{w}}}{8 L_{\mathrm{d}}^{3} \Omega_{0}^{2}}\left(1+K^{2} / 2\right)$

for $\lambda \approx \lambda_{\mathrm{w}}\left(1+K^{2} / 2\right) / 2 \gamma^{2}$ where $K$ is the wiggler parameter. For the present example, optimal performance requires that $\Delta E_{\mathrm{b}} / E_{\mathrm{b}}<0.01 \%$ for a $2 \mathrm{kG}$ field in the chicanes.

It is important to note that while the sensitivity to energy spread improves in a single-segment wiggler as the fundamental wavelength increases from 1.5 to $4.5 \AA$ with a decrease in beam energy, this is not necessarily the case in the optical klystron. The reason for this is contained in Eq. (1) where the change in path length through the chicane depends upon the product of $\gamma^{2} \lambda$ that is independent of beam energy. Thus, the principal advantage of the nonlinear harmonics is in a shorter saturation length. However, we could have reduced the sensitivity to energy spread if, instead of using lower beam energy to reach $4.5 \AA$, we had increased the wiggler period.

Our principal purpose is to examine harmonic performance for the generation of $1.5 \AA$ X-rays, and we begin by examining the fundamental performance. The power at the fundamental $(4.5 \AA)$ as a function of axial distance is shown in Fig. 2 for $\varepsilon_{n}=1.5 \pi \mathrm{mmmrad}$ and $\Delta E_{\mathrm{b}} / E_{\mathrm{b}}=0$, $0.001 \%$, and $0.002 \%$. The single-segment performance is also shown for comparison. In all cases, the initial noise power was chosen to be $226 \mathrm{~W}$. Note that saturation occurs over a distance of 


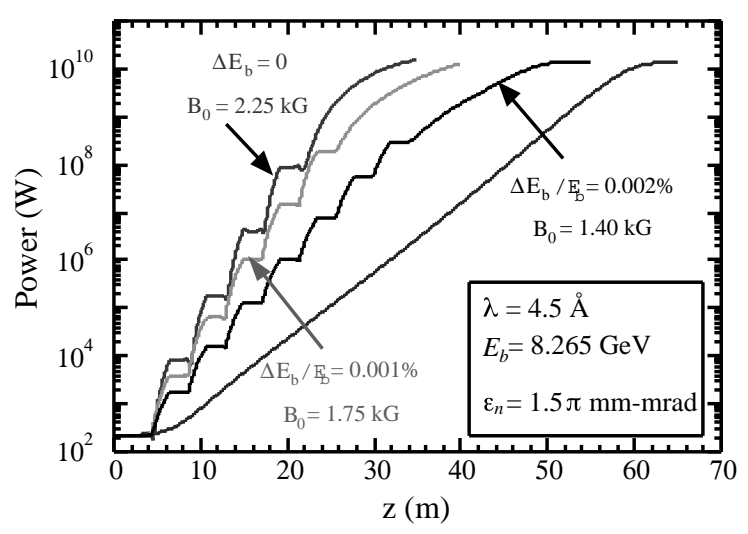

Fig. 2. Fundamental performance at $4.5 \AA$ for $\varepsilon_{n}=1.5 \pi \mathrm{mm}$ mrad.

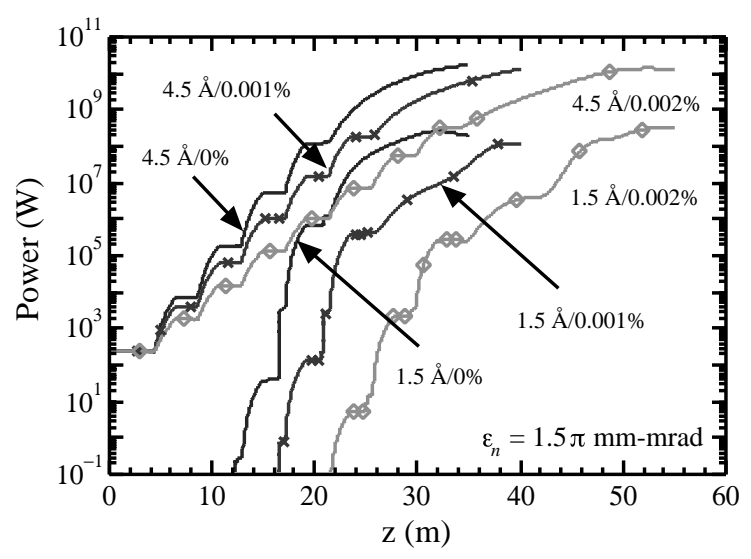

Fig. 3. Fundamental $(4.5 \AA)$ and harmonic $(1.5 \AA)$ performance for $\varepsilon_{n}=1.5 \pi \mathrm{mm}$ mrad.

approximately $65 \mathrm{~m}$ for the single-segment wiggler. In the ideal case of a vanishing energy spread, this distance can be reduced to a total length of about $35 \mathrm{~m}$ including six wiggler segments with a total length of only $26.25 \mathrm{~m}$. As the energy spread increases, the performance degrades; however, substantial advantages over the single-segment wiggler can still be obtained for energy spreads up to about $0.002-0.003 \%$.

We now turn to the harmonic performance. The harmonics are undriven in the simulations and grow due to the bunching at the fundamental [10]. The evolution of the fundamental and harmonics for the preceding example for $\varepsilon_{n}=1.5 \pi \mathrm{mm} \mathrm{mrad}$ is shown in Fig. 3. For $\Delta E_{\mathrm{b}} / E_{\mathrm{b}}=0$ the peak power at the fundamental is $16.95 \mathrm{GW}$ while the harmonic peaks at $265.5 \mathrm{MW}$ for a power ratio of the harmonic to the fundamental of $1.6 \%$. When the energy spread increases to $\Delta E_{\mathrm{b}} / E_{\mathrm{b}}=$ $0.001 \%$, the fundamental power drops to $12.76 \mathrm{GW}$ and the harmonic power to $124.2 \mathrm{MW}$ for a power ratio of $1.0 \%$. As the energy spread increases further to $\Delta E_{\mathrm{b}} / E_{\mathrm{b}}=0.002 \%$, the fundamental and harmonic powers reach $13.87 \mathrm{GW}$ and $310.6 \mathrm{MW}$, respectively, for a power ratio of $2.2 \%$. Note that the increased power at $\Delta E_{\mathrm{b}} / E_{\mathrm{b}}=$ $0.002 \%$ relative to $0.001 \%$ is due to the fact that it is difficult to precisely find the optimum magnetic field.

The sensitivity to the chicane magnetic field is also derived from Eq. (1), and has been noted in the previous studies [8,9]. It follows from Eq. (1) that, for the parameters of interest, $\Delta L / \lambda \approx 4.9 \Delta B_{0}$ for $\Delta B_{0}$ in Gauss and $B_{0} \approx 2 \mathrm{kG}$. The performance, therefore, should be roughly periodic for integer changes in $\Delta L / \lambda$, and this implies performance variations as the magnetic field changes by about $0.2 \mathrm{G}$. This is clearly shown in Fig. 4 , where we plot the fundamental and harmonic performance for $\Delta E_{\mathrm{b}} / E_{\mathrm{b}}=0$ and an emittance of $1.5 \pi \mathrm{mm}$ mrad near the optimal $B_{0}$. The performance for $1.0 \pi \mathrm{mm}$ mrad is similar, and the evolution of the power at the fundamental and harmonic for $\Delta E_{\mathrm{b}} / E_{\mathrm{b}}=0,0.002 \%$, and $0.004 \%$ is shown in Fig. 5.

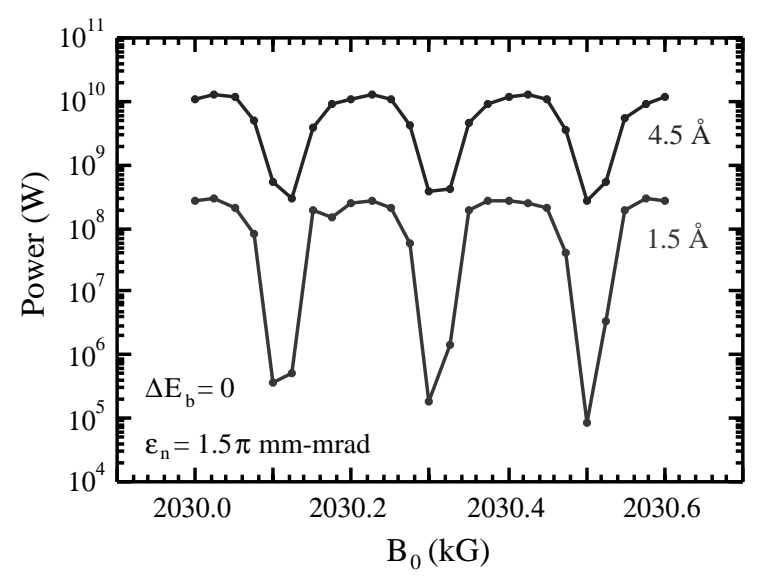

Fig. 4. Variation in the power in the fundamental and third harmonic with dipole field strength. 


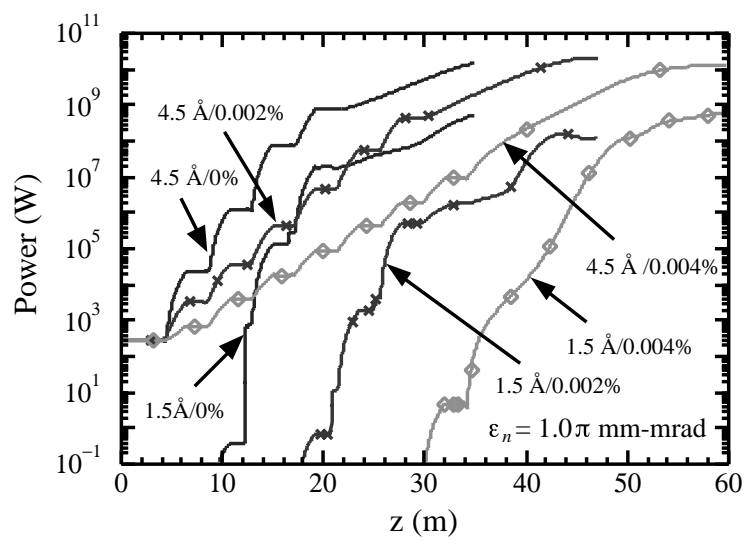

Fig. 5. Fundamental $(4.5 \AA)$ and harmonic $(1.5 \AA)$ performance for $\varepsilon_{n}=1.0 \pi \mathrm{mm}$ mrad.

In summary, we have presented a study of the performance of a distributed optical klystron operating on the $3 \mathrm{rd}$ harmonic to reach a wavelength of $1.5 \AA$. This has the advantage that faster than exponential growth greatly reduces the saturation length, while the principal disadvantage is the stricter constraint on beam quality. However, it should be remarked that while the energy spread required for an optical klystron to be effective at a wavelength of $1.5 \AA$ is below the currently projected slice energy spread $(0.006 \%)$ of the LCLS, the discrepancy is within a factor of only $2-3$. Hence, only a modest improvement in technology is required for this concept to be as effective as an alternative to the LCLS design.

\section{Acknowledgements}

Computational work was supported by the Advanced Technology Group at SAIC under IR\&D subproject 01-0060-73-0890-000. One of us (GRN) was supported by DOE Contract DEAC05-84ER40150 and the Commonwealth of Virginia.

\section{References}

[1] B. Faatz, et al., Nucl. Instr. and Meth. A 375 (1996) 441.

[2] NTIS Doc. No. DE98059292 (LCLS Design Group, "LCLS Design Report," April 1998), National Technical Information Service, Springfield, VA 22162.

[3] S.V. Milton, et al., Phys. Rev. Lett. 85 (2000) 988.

[4] N.A. Vinokurov, A.N. Skrinsky, preprint INP 77-59, Novosibirsk, 1977.

[5] V.N. Litvinenko, et al., Nucl. Instr. and Meth. A 304 (1991) 463.

[6] V.A. Bazylev, M.M. Pitatelev, Nucl. Instr. and Meth. A 358 (1995) 64

[7] N.A. Vinokurov, Nucl. Instr. and Meth. A 375 (1996) 264.

[8] G.R. Neil, H.P. Freund, Nucl. Instr. and Meth. A 475 (2001) 381, this issue.

[9] H.P. Freund, T.M. Antonsen Jr., Principles of Freeelectron Lasers, 2nd Edition, Chapman \& Hall, London, 1996.

[10] H.P. Freund, et al., IEEE J. Quantum Electron. 36 (2000) 275.

[11] M. Xie, Proceedings of IEEE 1995 Particle Accelerator Conference (IEEE Cat. No. 95CH35843), 1995, p. 183. 